\title{
The Value of a Modified Wiltse Approach for Deformity Correction in Neuromuscular Scoliosis
}

\author{
DAVID CHRISTOPHER KIESER, MBChB, PhD, FRACS, ${ }^{1}$ CHRISHAN THAKAR, MBChB, FRCS, ${ }^{2}$ \\ GREGORY CUNNINGHAM, FRACS, ${ }^{2}$ HRVOJE VIDAKOVIC, MBChB, PGDipSurgAnat, ${ }^{1}$ NIELS HAMMER, \\ $\mathrm{MD},{ }^{3}$ COLIN NNADI, MBBS, FRCSI, FRCS ${ }^{2}$ \\ ${ }^{I}$ Department of Orthopaedic Surgery and Musculoskeletal Medicine, University of Otago, Christchurch School of Medicine, Christchurch, New Zealand, \\ ${ }^{2}$ University of Oxford, Division of Spinal Surgery, Oxford, England, ${ }^{3}$ Department of Anatomy, University of Otago, Dunedin School of Medicine, Dunedin, New \\ Zealand
}

\begin{abstract}
Background: Wiltse approaches have been shown to reduce operative blood loss and enhance recovery in lumbar spinal surgery; however, their efficacy in neuromuscular scoliosis (NMS) deformity correction has never been assessed. Thus, the purpose of this study was to compare the outcomes of deformity correction requiring pelvic fixation in NMS performed through a Wiltse approach in contrast to a standard midline approach.

Methods: This is a retrospective review of 24 consecutive children with NMS undergoing deformity correction by a single surgeon in our institution. Patient demographic data, operative time, blood loss, curve correction, length of stay, and complications were recorded.

Results: In 16 children, the procedure was performed through a Wiltse approach and in 8 through a midline approach. There was no significant difference in age, sex, preoperative Cobb angle, or number of levels fused. Patients who underwent a Wiltse approach had significantly less blood loss yet similar curve correction. The hospital length of stay and complication rate were not significantly different between the groups.

Conclusions: A modified Wiltse approach can be safely used for NMS deformity correction. This approach achieves similar curve corrections to a traditional midline approach with less blood loss and no need for iliac screw connectors.
\end{abstract}

Level of Evidence: 3.

Lumbar Spine

Keywords: scoliosis, spine, spinal fusion, neuromuscular, iliac screw, Wiltse

\section{INTRODUCTION}

Neuromuscular scoliosis (NMS) is a challenging condition to treat and often progresses with nonoperative care. ${ }^{1,2}$ Operative intervention is therefore commonly considered in order to align the spine for optimal internal organ physiology as well as ease of personal care with symmetrical loading in an erect posture. ${ }^{1,3}$ Because of the poor motor control related to the underlying condition, the large number of vertebrae involved in the scoliosis, and the frequency of pelvic obliquity, rigid fixation extending to the pelvis is usually warranted. ${ }^{1}$

Multiple options exist for pelvic fixation, including sacral screws, sacroiliac screws, and iliac screws. ${ }^{4}$ Sacral screws offer multiple fixation points and the potential for bicortical fixation. In addition, the screw heads align with the pedicle screws of the spine, negating the need for connectors. However, sacral screws do not directly disperse the load into the surrounding pelvis and therefore risk failure due to overcoming of the constructs biomechanical stability or adjacent sacroiliac joint disease. ${ }^{5,6}$

In order to disperse the load and increase the screw length, size, and strength, sacroiliac screws or ilio-sacral bolts can be used. These maintain the screw heads in-line with the spinal pedicle screws but can be challenging to insert and transfix the sacroiliac joint, reducing pelvic micromotion. Iliac screws offer an attractive alternative to increase the dispersion of the biomechanical load to the pelvis without violating the sacroiliac joint. ${ }^{7}$ However, these screws lie lateral to the alignment of spinal pedicle screws, are challenging to insert, and may be prominent, thus causing metalware irritation. ${ }^{8}$

Deformity correction extending to the pelvis with the use of iliac screws is most commonly performed through a midline approach with elevation of the erector musculature from the posterior iliac brim 
and posterior spinal elements to allow access for screw insertion. However, midline approaches involve significant soft tissue mobilization that risks increased blood loss, surgical site infection, and posterior musculature denervation; therefore, a Wiltse technique has been advocated for posterior spinal fixation. ${ }^{9}$ In addition, midline posterior approaches in children with posterior element deficiencies, often identified in NMS, risk neurological or dural injury; thus, more lateral Wiltse approaches are presumed to be safer.

We have adopted a modified Wiltse technique for deformity correction in children with NMS in the belief that it has a lower complication profile with respect to soft tissue injury, posterior musculature function, midline neural injury, and operative blood loss while still achieving satisfactory deformity correction without an increase in complication profile. This study compares the outcome of our patients with NMS who have undergone deformity correction through a modified Wiltse technique and a standard midline technique.

\section{METHODS}

We performed a retrospective review of 24 consecutive children with NMS undergoing deformity correction by the senior author $(\mathrm{CN})$ from the upper thoracic spine to the pelvis. Institutional review board approval was obtained, but patient consent was not felt necessary because of the retrospective nature of this study. Inclusion criteria were all children, under the age of 18 years, undergoing deformity correction with a diagnosis of NMS. This was a consecutive series, and our only exclusion criterion was a minimum 1-year followup. Of the 24 consecutive children included, the first 8 underwent a midline approach, as this was initially our preferred approach. The subsequent 16 underwent deformity correction using a Wiltse approach. We compared the operative time, estimated blood loss (EBL), deformity correction, hospital length of stay (LOS), and complication risks between those undergoing a modified Wiltse approach and those with a midline approach.

The patient weight and age (defined as age in years) was recorded at the time of the procedure. Similarly, their Gross Motor Function Classification System, vocalization, and gastric feeding requirements were recorded immediately prior to surgery. The Cobb angle was defined as the major coronal Cobb angle, which was recorded preoperatively and at final follow-up. Similarly, the pelvic incidence, sacral slope, lumbar lordosis, thoracic kyphosis, and spino-sacral angle were recorded preoperatively and at final follow-up. The operative time was recorded at the time of the procedure from the time of the incision until completion of the wound closure and application of the dressings. EBL was recorded by a blood transfusion expert from the suction volume and weighing of swabs. The time, in days, in the intensive care unit (ICU) was attained from the notes, as was the hospital (LOS and complications.

\section{Surgical Approach}

All patients underwent a general anesthetic with neuromonitoring and were positioned prone on bolsters. All patients received weight-specific doses of tranexamic acid and intrathecal morphine. A midline skin incision was used in all patients. After the respective approaches, the facet joints included in the fusion were excised to enhance fusion. Pedicle screws were inserted using standard anatomical landmarks. Bilateral L5 pedicle screws were used in all cases. Once the pedicle screws were inserted, we inserted the iliac screw. We did not utilize intraoperative navigation, only fluoroscopy; therefore, palpation was used to guide the iliac screw trajectory by sliding the index finger over the thickened outer table of the lower ilium. Our entry point was deliberately positioned low on the posterior-superior iliac spine (PSIS) to ensure that the screw head was not prominent. We utilized Solera instrumentation and the Ballast iliac screw (Medtronic Ltd, Dublin, Ireland) because the screw head is flush, and we felt this was less likely to cause soft tissue irritation. We utilized 2 6.0-mm titanium rods, which were connected with a cross connector to enhance stability. Wounds were closed in layers with vicryl to the iliolumbar fascia and subcutaneous tissue and monocryl to the skin. Dry dressings were applied without negative pressure therapy. No plastic surgical input was utilized.

\section{Modified Wiltse Approach (Figure 1)}

After a midline skin incision, a modified bilateral Wiltse approach was used to expose the PSIS, facet joints, and pars of the lumbar spine through a plane between multifidus and longissimus. The distal border of the plane is the PSIS, which was exposed by elevating the periosteum from the inner and outer tables of the ilium. A standard midline dissection (described below) was used in the mid- 


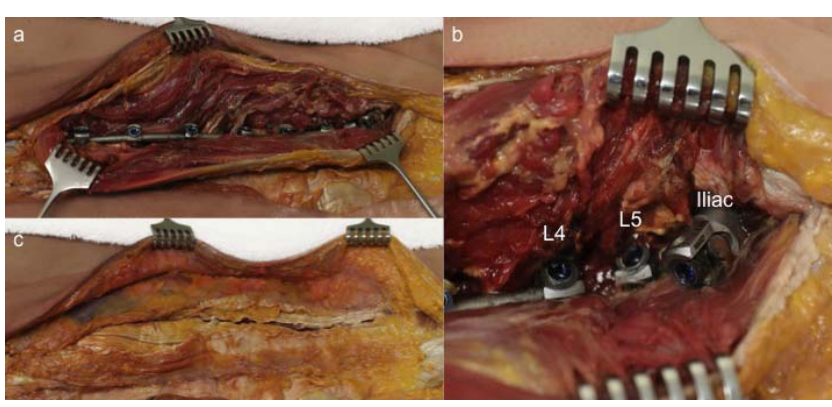

Figure 1. Selected images of a cadaveric example with a unilateral Wiltse approach for insertion of ilio-lumbar fixation. Image (a) illustrates the metalware in situ with image (b) illustrating the ilio-lumbar fixation. Image (c) shows soft tissue apposition and ease of coverage of the instrumentation, including the iliac screw.

and upper-thoracic spine to avoid incision of the latissimus dorsi, trapezius, and peri-scapular musculature necessary with a para-midline incision. After facet preparation, screw insertion was performed. The rod was then inserted by submuscular tunneling between the Wiltse and midline incision. Deformity correction was performed, and then the thoraco-dorsal fascia incisions, including the insertion onto the iliac crest, were closed. The subcutaneous fat and midline skin incisions were then closed without a drain.

\section{Midline Approach}

After the skin incision, the thoraco-dorsal fascia was elevated from the spinous processes and the paraspinal musculature elevated from the posterior spine to fully expose the facet joints. The PSIS was exposed by elevation of the posterior musculature. After facet preparation, screw and rod insertion, and deformity correction, we repaired the thoraco-dorsal fascia incision. The subcutaneous fat and midline skin incisions were then closed without a drain.

\section{Statistical Analysis}

Statistical analysis was performed using SPSS software (IBM Corp, Armonk, NY). The MannWhitney $U$ test and Fisher exact test were used to compare demographic and operative data, including curve correction. A $t$ test was used to compare preand postoperative curve parameters as well as the mean complication rate of the 2 groups. A $P$ value $<.05$ was considered to be statistically significant.

\section{RESULTS}

There were no children excluded from the study. The comparative demographics and curve severity between the groups are shown in Table 1. No statistically significant differences were identified
Table 1. Demographic and curve comparison between the groups (mean with standard deviation in parentheses).

\begin{tabular}{|c|c|c|c|}
\hline & Midline & Wiltse & $P$ Value \\
\hline No. of patients & 8 & 16 & \\
\hline Age $(y)$ & $14.7(2.8)$ & $14.0(3.5)$ & .606 \\
\hline Sex & 5 male, 3 female & 8 male, 8 female & 1.00 \\
\hline Weight (kg) & $38.1(18.0)$ & $35.5(12.8)$ & .725 \\
\hline \multicolumn{4}{|l|}{ GMFCS } \\
\hline 3 & 1 & 2 & \\
\hline 4 & 2 & 5 & \\
\hline 5 & 5 & 8 & \\
\hline Nonverbal & 2 & 7 & .648 \\
\hline $\begin{array}{l}\text { Nasogastric tube or } \\
\text { gastrostomy }\end{array}$ & 3 & 10 & .376 \\
\hline Pelvic incidence $\left(^{\circ}\right)$ & $45.6(10.7)$ & $46.2(14.2)$ & .972 \\
\hline Sacral slope $\left(^{\circ}\right)$ & $34.9(24.6)$ & $21(28.2)$ & .436 \\
\hline Lumbar lordosis $\left(^{\circ}\right)$ & $37.6(31.5)$ & $36.8(36.5)$ & .934 \\
\hline Thoracic kyphosis $\left(^{\circ}\right)$ & $27.9(13.5)$ & $36.1(22.2)$ & .481 \\
\hline Major Cobb angle $\left(^{\circ}\right)$ & $82.1(23.8)$ & $87.1(23.0)$ & .623 \\
\hline Spino-sacral angle $\left(^{\circ}\right)$ & $-3.0(35.6)$ & $10.0(18.2)$ & .115 \\
\hline \multicolumn{4}{|l|}{ Follow-up (mo) } \\
\hline Mean & $85.2(23.8)$ & $32.0(14.0)$ & .085 \\
\hline Range & $53-119$ & $16-65$ & \\
\hline
\end{tabular}

between the groups. All patients in the midline group had cerebral palsy (CP). In the Wiltse group, 11 had CP, 1 had Rett syndrome, 1 had cortical dysplasia, 1 had spina bifida, and 1 had an inverted chromosomal duplication of $8 \mathrm{P}$.

The procedural details are shown in Table 2. The number of levels incorporated and the LOS in both the ICU and the hospital were similar. A reduced operative time was found for the Wiltse technique; however, this was not statistically significant. The EBL was significantly less with a Wiltse approach $(P$ $=.001)$. No connectors were required between the iliac screw and rod in the Wiltse approach, but connectors were always used in the midline approach.

The surgical correction and complications are shown in Table 3 . The curve correction was similar between the groups $(P=.496)$ (Figure 2). Four patients in the Wiltse group and 4 in the midline group experienced a surgical complication $(P=$ .134). No patients in the Wiltse group had prominent metalware, but 3 experienced an infection, and 1 had a rod breakage. In contrast, 1 patient had metalware prominence; 2 experienced an infection and 1 developed adjacent segment disease in those with a midline approach.

\section{DISCUSSION}

This study is the first to describe a modified Wiltse technique and compare it to a standard midline approach for spinal deformity correction in children with NMS. Our results have shown a reduced blood loss with equivalent curve correction 
Table 2. The procedural and perioperative comparison between the groups (mean with standard deviation in parentheses).

\begin{tabular}{lcccc}
\hline & Number of Levels & Operative Time & $\begin{array}{c}\text { Estimated Blood } \\
\text { Loss }(\mathbf{m L})\end{array}$ & $\begin{array}{c}\text { Intensive Care Unit } \\
\text { Length of Stay (d) }\end{array}$ \\
\hline Wiltse & $16.2(0.41)$ & $296.9(57.3)$ & $795.3(370.5)$ & $3.0(1.5)$ \\
Midline & $15.8(0.46)$ & $340.9(79.7)$ & $2438.9(1635.7)$ & $2.3(1.6)$ \\
$P$ value & .131 & .169 & .001 & .367 \\
\hline
\end{tabular}

and complication rates. Furthermore, the operative time, although not statistically significant, was less in the Wiltse technique despite more levels being incorporated in this group.

We initially used a standard midline approach in the first 8 children because this was the preferred technique at the time. However, at the end of 2014, we elected to change our approach to a modified Wiltse approach because we felt that the reduced muscle elevation could improve early functional outcome and LOS. In comparing the groups, we feel that they are comparable, with no significant difference in age, sex, curve severity, and number of levels fused. However, because of the distinct time point for our change in surgical approach, patients with a midline incision had a longer follow-up.

Overall, we found a reduced operative time and significantly reduced blood loss in patients undergoing a modified Wiltse technique. This is consistent with previous authors who have identified a reduced blood loss in adult spinal fusions with a Wiltse technique and is believed to be because the Wiltse approach is an intermuscular avascular plane. ${ }^{9,10}$

Similarly, because this approach is muscle sparing, previous authors have proposed that it enhances recovery and reduces hospital LOS. ${ }^{10,11}$ Our results did not demonstrate a significant difference in either ICU or hospital LOS. Further research with greater patient numbers is warranted in order

Table 3. Curve correction and complication comparison between the groups (mean with standard deviation in parentheses).

\begin{tabular}{lccc}
\hline & Midline & Wiltse & P Value \\
\hline Postoperative Cobb angle $\left(^{\circ}\right)$ & $52.3(20.9)$ & $61.8(22.1)$ & .319 \\
${\text { Cobb correction }\left(^{\circ}\right)}{ }^{\circ}$ & $29.8(12.5)$ & $25.3(16.3)$ & .496 \\
Sacral slope $\left(^{\circ}\right)$ & $34.8(18.0)$ & $28.5(18.0)$ & .434 \\
Lumbar lordosis $\left(^{\circ}\right)$ & $64.5(22.0)$ & $51.3(24.1)$ & .21 \\
Thoracic kyphosis $\left(^{\circ}\right)$ & $31.8(17.1)$ & $38.6(14.8)$ & .331 \\
Spino-sacral angle $\left(^{\circ}\right)$ & $1.6(14.5)$ & $1.9(14.2)$ & .962 \\
Complication & & & \\
Infection & 2 & 3 & \\
Metalware prominence & 1 & 0 & \\
Rod breakage & 0 & 1 & \\
Adjacent segment disease & 1 & 0 & \\
Symptomatic seroma & 0 & 0 & \\
Pseudarthrosis & 0 & 0 & \\
Screw loosening & 0 & 0 & \\
Reoperations & 2 & 3 & \\
\hline
\end{tabular}

to identify whether there is a significant difference in these outcomes.

The overall complication rate was halved in the Wiltse group compared to the midline group, although this may be attributed to the longer follow-up in the midline group. Infection was the most common complication, consistent with previous reports of deformity correction in NMS. ${ }^{12}$ No significant difference in infection rate was identified between the groups. Because no patients experienced a neurological injury in either group, we cannot confirm that a Wiltse technique is useful in patients with spinal dysraphism and posterior element defects, where a midline approach may endanger the unprotected neural elements. In addition, this approach allows a more oblique pedicle screw entry that ensures that the screw heads, including the iliac screw, line up and therefore do not require rod connectors.

No patients had prominent metalware in the Wiltse technique group in contrast to 1 in the midline group. We feel that the iliac screw insertion point and subsequent screw head position can be equally placed through a Wiltse or midline approach but advocate an anterior starting position to ensure that the head is not prominent. Previous authors have suggested that 2 iliac screws per hemi-pelvis provides more rigid fixation and fewer implant failures. ${ }^{13}$ We have not assessed this technique but feel that the addition of a

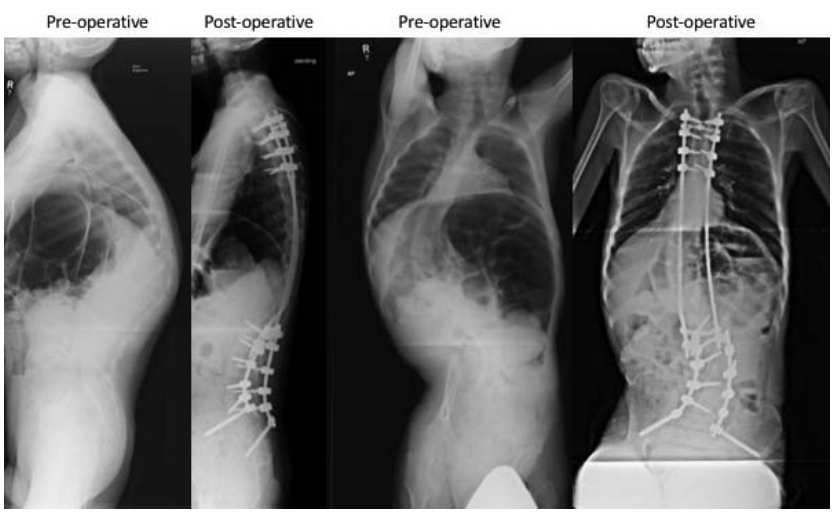

Figure 2. A representative case of a 35-kg male with a Gross Motor Function Classification System (GMFCS) 3 neuromuscular scoliosis who underwent a T3-pelvis fusion through a Wiltse approach. Preoperative and 2-year postoperative radiographs are shown. 
second iliac screw, if required, is easily inserted through either approach.

We used a midline incision in all patients in case of a future revision. However, the Wiltse approach requires undermining of the adipose tissue along the thoraco-dorsal fascia. Although none of our patients developed a hematoma or seroma, this remains a concern, and consideration of paramedian skin incisions should be given.

This study has a number of limitations. First, it is limited by its small numbers, which we feel relates to the lack of statistical significance in some of the defined outcome measures, particularly hospital LOS. Second, the multitude of conditions causing NMS and the variance in curve types further limit the interpretation of these results. Third, we analyzed a single surgeon's results to limit surgeon variance, but further research is necessary to validate this approach by others. Fourth, the shorter follow-up in the Wiltse group may confound complication outcomes. This is particularly of concern with definitive fusion, where the Wiltse technique limits the surface area for bony fusion. However, at a minimum 16-month follow-up, there remain no cases of symptomatic nonfusion. Finally, radiological follow-up was based on x-rays, which limits our capacity to determine definitive fusion.

In conclusion, a modified Wiltse approach can be safely used for NMS deformity correction. This approach achieves similar curve corrections to a traditional midline approach but with less blood loss and no need for iliac screw connectors.

\section{ACKNOWLEDGMENTS}

Glynny Kieser for her editorial input and Prof Chris Frampton for his statistical input and Medtronic for lending their cadaveric spinal instrumentation for the illustrative purposes of Figure 1.

\section{REFERENCES}

1. McCarthy RE. Management of neuromuscular scoliosis. Orthop Clin North Am. 1999;30:435-449.

2. Olafsson Y, Saraste H, Al-Dabbagh Z. Brace treatment in neuromuscular spine deformity. J Pediatr Orthop. 1999;19:376379.

3. Banta JV, Drummond DS, Ferguson RL. The treatment of neuromuscular scoliosis. Instr Course Lect. 1999;48:551-562.

4. Emami A, Deviren V, Berven S, Smith JA, Hu SS,
Bradford DS. Outcome and complications of long fusions to the sacrum in adult spine deformity: luque-galveston, combined iliac and sacral screws, and sacral fixation. Spine. 2002;27:776786.

5. Ha KY, Lee JS, Kim KW. Degeneration of sacroiliac joint after instrumented lumbar or lumbosacral fusion: a prospective cohort study over five-year follow-up. Spine 2008;33:11921198.

6. Katz V, Schofferman J, Reynolds J. The sacroiliac joint: a potential cause of pain after lumbar fusion to the sacrum. $J$ Spinal Disord Tech. 2003;16:96-99.

7. Peelle MW, Lenke LG, Bridwell KH, Sides B. Comparison of pelvic fixation techniques in neuromuscular spinal deformity correction: Galveston rod versus iliac and lumbosacral screws. Spine. 2006;31:2392-2399.

8. Ilyas H, Place H, Puryear A. A comparison of early clinical and radiographic complications of iliac screw fixation versus S2 alar iliac (S2AI) fixation in the adult and pediatric populations. J Spinal Disord Tech. 2015;28:E199-E205.

9. Street JT, Andrew Glennie R, Dea N, et al. A comparison of the Wiltse versus midline approaches in degenerative conditions of the lumbar spine. J Neurosurg. 2016;25:332-338.

10. Karikari IO, Isaacs RE. Minimally invasive transforaminal lumbar interbody fusion: a review of techniques and outcomes. Spine. 2010;35:S294-S301.

11. Anand N, Baron EM, Bray RS Jr. Benefits of the paraspinal muscle-sparing approach versus the conventional midline approach for posterior nonfusion stabilization: comparative analysis of clinical and functional outcomes. SAS J. 2007;1:93-99.

12. Nandyala SV, Schwend RM. Prevalence of intraoperative tissue bacterial contamination in posterior pediatric spinal deformity surgery. Spine. 2013;38:E482-E486.

13. Phillips JH, Gutheil JP, Knapp DR Jr. Iliac screw fixation in neuromuscular scoliosis. Spine. 2007;32:1566-1570.

Disclosures and COI: The authors report no conflict of interest concerning the materials or methods used in this study or the findings specified in this article.

Corresponding Author: David Kieser, MBChB, PhD, FRACS, Department of Orthopaedic Surgery and Musculoskeletal Medicine, Canterbury District Health Board, 2 Riccarton Avenue, Christchurch 8011, New Zealand. Phone: 006421 149 9829; Fax: 00643467 9709; Email: kieserdavid@gmail.com.

Published 30 April 2020

This manuscript is generously published free of charge by ISASS, the International Society for the Advancement of Spine Surgery. Copyright (C) 2020 ISASS. To see more or order reprints or permissions, see http://ijssurgery.com. 